\title{
Role of Depression and Anxiety Disorders in Takotsubo Syndrome: The Psychiatric Side of Broken Heart
}

Federico Oliveri ${ }^{1}$, Harshit K. Goud ${ }^{2}$, Lubna Mohammed ${ }^{3}$, Zainab Mehkari ${ }^{2}$, Moiz Javed ${ }^{2}$, Aldanah Althwanay ${ }^{2}$, Farah Ahsan ${ }^{2}$, Ian H. Rutkofsky ${ }^{4}$

1. Cardiology, California Institute of Behavioral Neurosciences \& Psychology, Fairfield, USA 2. Internal Medicine, California Institute of Behavioral Neurosciences \& Psychology, Fairfield, USA 3. Obstetrics \& Gynaecology, California Institute of Behavioral Neurosciences \& Psychology, Fairfield, USA 4. Psychiatry, California Institute of Behavioral Neurosciences \& Psychology, Fairfield, USA

Corresponding author: Federico Oliveri, federico.md.oliveri@gmail.com

\begin{abstract}
Takotsubo syndrome (TTS), also called broken heart syndrome, is an acute and transient cardiac wall motion abnormality of the left ventricle. The patient prototype is a post-menopausal woman with myocardial infarction-like symptoms (angina pectoris, breathlessness, palpitations, etc.) who has experienced sudden emotional or physical stress. Although prognosis is generally considered relatively benign, both complications and recurrence rates are not insignificant. Pathophysiological mechanisms underlying TTS are not entirely understood, but the sympathetic system over-activity has a leading role. Moreover, since emotional stress frequently triggers TTS and since precedent diagnosis of psychiatric disorders sometimes coexists, the psychological response to stress could be another potential therapeutic target. Indeed, this article aims to explore the association between underlying depression and anxiety disorders and TTS, as well as to find ideal therapeutic options useful to treat and prevent TTS. Thus in our review, we considered case reports, case-control studies, and review articles from PubMed. Papers dealing with Takotsubo syndrome and anxiety disorder or depression were selected. We included papers published since 2010 and whose abstract was in English. We concluded that anxiety disorders, but not depression, are associated with a higher occurrence of TTS. There is a link between anxiety, TTS, and inflammation leading to increased sympathetic activity. Nevertheless, patients with pre-admission psychiatric disorders have a higher risk of recurrent TTS. Importantly, the use of selective serotonin reuptake inhibitors (SSRIs) could be a potential therapeutic aid in preventing TTS's recurrence in selected patients.
\end{abstract}

Categories: Cardiology, Internal Medicine, Psychiatry

Keywords: takotsubo syndrome, psychiatric disorders, anxiety disorders, depression

Received 08/13/2020

Review began 08/29/2020 Review ended 09/07/2020 Published 09/11/2020

() Copyright 2020 Oliveri et al. This is an open access article distributed under the terms of the Creative Commons Attribution License CC-BY 4.0., which permits unrestricted use, distribution, and reproduction in any medium, provided the original author and source are credited.

\section{Introduction And Background}

Takotsubo syndrome (TTS), also called apical ballooning syndrome (ABS), broken heart syndrome, or stress cardiomyopathy, is a syndrome characterized by transient regional systolic dysfunction of the left ventricle (LV), resembling myocardial infarction (MI), but in the absence of angiographic evidence of acute plaque rupture or hemodynamically significant obstructive coronary artery disease (CAD) [1]. Although there are different subtypes of regional systolic disfunction, the "classical" anatomical pattern of TTS is characterized by left ventricle regional wall motion abnormality with apical and circumferential mid-ventricular hypokinesia and basal hyperkinesia. At end-systole, the left ventricle typically resembles the 'Takotsubo' (Japanese name used to indicate an octopus pot) [2], with a narrow neck and larger lower portion, giving the appearance of apical ballooning [3,4]. Two out of 10,000 hospitalizations $(0.02 \%)$ in the United States are considered caused by TTS [5].

The majority of patients (89-91\%) are elderly post-menopausal women (66-80 years of age), while the overall mean age is $66.9 \pm 30.7$ years and most patients $(59.6 \%)$ are $\geqslant 65$ years old [4-6]. Indeed the typical patient with primary TTS is a post-menopausal woman who has experienced severe, sudden emotional or physical stress [4]. It can happen in a background of high personal stress or, in some cases, in patients with a previous diagnosis of psychiatric disorders $[5,7]$. However, the mortality rate is higher in men $(8.4 \%$ vs. $3.6 \%$ ), probably reflecting the higher frequency of underlying severe critical illness (36.6\% in men vs. $26.8 \%$ in women) [6]. Patients with TTS typically present with acute angina pectoris, breathlessness, palpitations, but also ventricular tachyarrhythmias and/or cardiogenic shock may be present [4]. Diagnosis of Takotsubo syndrome is usually made following the Mayo Clinic diagnostic criteria, all four of which are required [8]: I. Reversible (transient) left ventricular systolic (LV) dysfunction (dyskinesis, hypokinesis, akinesis). Left ventricle regional wall motion abnormality extends beyond a single epicardial vascular distribution; II. Absence of significant obstructive coronary artery disease $(C A D)$ or angiographic evidence of acute plaque rupture. If CAD is present, the diagnosis of TTS can still be made if the wall motion abnormalities extend beyond a single epicardial vascular distribution; III. New ECG abnormalities (either T-wave inversion or ST-segment elevation) or modest elevation in cardiac troponin; IV. Absence of myocarditis or pheochromocytoma. 


\section{Cureus}

Although Takotsubo syndrome is generally considered having a relatively benign prognosis with rapid recovery of LV function, newer evidence suggests TTS is a more acute severe cardiac disorder with a variety of complications in $\sim 52 \%$ of patients [9-11]. Systolic heart failure is the most common complication in the acute phase of Takotsubo syndrome, occurring in $12-45 \%$ of cases [10-12]. Ventricular arrhythmias occur in $4-9 \%$ of patients during the acute phase of the syndrome, causing cardiac arrest in $4-6 \%$ of cases [13-15]. The in-hospital mortality rate is $2-5 \%$, mainly due to ventricular fibrillation or refractory cardiogenic shock $[5,6,9-11]$. The pathophysiology of Takotsubo syndrome is complex and not fully understood but catecholamines appear to play a pivotal role as the trigger is often sudden unexpected stress [4]. Five-year recurrence rates of $5-22 \%$ have been reported, with the second episode occurring three months to 10 years after the first $[4,16]$. Preventive therapy of recurrence has yet to be found. Intuitively, beta-blockers may provide some protection against future catecholamine surges. However, recurrences have been reported in patients taking beta-blockers, and meta-analysis found no impact of these medications on the risk of recurrence [17]. Given the high frequency of stressful triggers in cases of TTS [4], the psychological response to stress could be another potential therapeutic target. Indeed, since both mortality and recurrence rates are significant $[4,6,16]$, this review aims to explore the possible association between underlying psychiatric disorders and TTS. Nevertheless, by better understanding the pathophysiological mechanisms underlying TTS, potential useful therapeutic targets could be found to reduce both the onset and recurrence of Takotsubo syndrome.

\section{Review}

Method

Research Process

The research procedure was conducted in two steps. Firstly we have searched articles about risk factors, pathophysiology, treatment, and recurrence prevention of Takotsubo syndrome; then, we combined Takotsubo and psychiatric disorders, selecting articles that deal with depression and anxiety disorders in patients with coexisting TTS. We used PubMed and Google Scholar as primary electronic databases to find materials. Keywords used for our process were: Takotsubo syndrome, psychiatric disorders, anxiety disorders, depression. Results are presented as follow (Table 1):

\begin{tabular}{|l|c|}
\hline KEYWORD/COMBINATION OF KEYWORDS & NUMBER OF RESULTS \\
\hline Psychiatric disorders & 213902 \\
\hline Depression & 123969 \\
Anxiety & 3923 \\
\hline Takotsubo syndrome & 155 \\
\hline Takotsubo syndrome and psychiatric disorders & 147 \\
\hline Takotsubo syndrome and depression & 72 \\
\hline Takotsubo syndrome and anxiety & 2 \\
\hline
\end{tabular}

TABLE 1: Keywords and Number of Articles Found

Case reports, case-control studies, and review articles were considered in our review. Papers dealing with Takotsubo syndrome and anxiety disorder or depression were included. We selected publications whose abstract was in English, while papers published before 2010 were not considered. No geographic considerations were given.

\section{Discussion}

Pre-Existing Psychiatric Disorders and TTS

To date, both depression and anxiety disorders are associated with increased cardiovascular disease, events, and death [18-20]. Specifically, anxiety is perhaps considered the most critical risk factor for cardiovascular disease [21]. Less evidence regarding the possible association between psychiatric disorders, specifically anxiety disorder and depression, and Takotsubo syndrome is available. A retrospective study compared TTS patients taken by the International Takotsubo Registry with 455 sex- and age-matched patients with a diagnosis of acute coronary syndrome (ACS). This study was conducted to investigate epidemiological 
characteristics, clinical features, prognostic predictors, and outcomes of TTS. The analysis showed that total psychiatric disorders, both acute and chronic, were statistically more frequent in patients with TTS than those with ACS; past or chronic anxiety disorders were statistically more prevalent in the TTS group than the ACS group; and there was no statistically prevalence difference in acute anxiety disorders between the two groups [22] (Table 2).

\begin{tabular}{|c|c|c|c|c|c|}
\hline PSYCHIATRIC DISORDER & TTS (n) & TTS (\%) & $\operatorname{ACS}(n)$ & ACS (\%) & P-VALUE \\
\hline Total (acute+chronic) & 191 & 42,3 & 64 & 14,3 & $<0,001$ \\
\hline Acute psychiatric disorder & 57 & 12,6 & 6 & 1,3 & $<0,001$ \\
\hline Acute anxiety disorder & 4 & 0,9 & 0 & 0,0 & 0,120 \\
\hline Past or chronic psychiatric disorders & 165 & 36,6 & 61 & 13,6 & $<0,001$ \\
\hline Past or chronic anxiety disorders & 45 & 10,0 & 4 & 0,9 & $<0,001$ \\
\hline
\end{tabular}

TABLE 2: Comparison of the Prevalence of Psychiatric Disorders in Patients With TTS and ACS

TTS: Takotsubo syndrome, ACS: acute coronary syndrome

It should be noted that the authors did not analyze "depression” alone as a separate condition [22]. Therefore the possibility that depression had been more prevalent in one group or another cannot be affirmed or excluded.

In another retrospective case-control study, the authors assessed past psychiatric history preceding the episode of ABS in 25 TTS patients by comparing them with two group controls. The first control group was based on 25 age- and sex-matched patients with previous ST-elevation myocardial infarction (STEMI), while the second one included 50 age- and sex-matched patients from the general population (GP) [23]. The results showed that chronic anxiety disorders were statistically more frequent in patients with Takotsubo syndrome group than the other groups, and depression did not occur with statistically significant differences between the three groups (Table 3).

\begin{tabular}{|c|c|c|c|c|}
\hline PSYCHIATRIC DISORDER & TTS (\%) & STEMI (\%) & GP (\%) & P-VALUE \\
\hline Anxiety or depression & 68 & 36 & 30 & $<0,050$ \\
\hline Chronic Anxiety disorders & 56 & 12 & 18 & $<0,001$ \\
\hline Depression & 48 & 28 & 22 & $>0,050$ \\
\hline
\end{tabular}

\section{TABLE 3: Comparison of the Prevalence of Psychiatric Disorders in Patients With TTS, STEMI,} and GP

TTS: Takotsubo syndrome, STEMI: ST-elevation myocardial infarction, GP: general population

So the previous study confirmed the higher prevalence of pre-morbid chronic anxiety disorders in TTS. Still, it does not highlight a statistically significant difference in depression disorders between the three groups. This study's strength is the use of two control groups (STEMI and GP), while its limitations are small sample size and non-multi-centric [23].

Another case-control study based on women with TTS confirms that compared to myocardial infarction (MI) and general healthy $(\mathrm{GH})$ controls, the pre-admission prevalence of anxiety disorders is statistically more prevalent in patients who had developed TTS than in the other two groups (24.4\% in TTS, 9.4\% in MI, and 0 in GH with $\mathrm{p}=0.007$ ). Moreover, this study has also shown that depression was not associated with the occurrence of TTS [24]. Since more than $90 \%$ of patients diagnosed with TTS are women [4-6], the sample based on women only is a relative limitation. A further study conducted on a huge number of patients with previous diagnoses of TTS $(n=24,701)$ confirmed the higher prevalence of anxiety disorders in this group compared to MI and orthopedic controls [25]. 


\section{Cureus}

a case-control study, 50 TTS patients and sex- and age-matched STEMI controls were asked to complete, during the hospitalization, a survey that measured the self-reported trait anxiety. A high-anxiety trait was a common finding, but there was not a statistically significant difference between the two groups [26].

Nevertheless, it should also be considered that the survey was submitted during the hospital stay, possibly being influenced by hospitalization-related anxiety. Another case-control study based on 19 patients with TTS and sex-, age-, and residence-matched controls has shown that only major depressive disorder (MDD) had a significantly higher prevalence in Takotsubo patients than controls [27]. The main limitations of the previous two above studies were the small sample size.

Indeed, by comparing the aforementioned studies, we are comfortable stating that anxiety, more precisely chronic anxiety disorders, is related to the occurrence of Takotsubo syndrome [22-25]. On the other hand, depression does not appear to be more prevalent in TTS patients [23,24].

TTS Triggers in Patients With and Without Pre-Admission Anxiety Disorders

The typical patient with primary TTS is a post-menopausal woman who has experienced severe, unexpected emotional or physical stress $[4,28]$. Some authors have recently described that in patients with pre-

admission anxiety disorders, TTS was predominantly triggered by exclusively emotional stress. In this study, triggering events were compared in 58 TTS patients with (group1) and without (group2) pre-existing anxiety disorders. The results showed that in patients with a past medical history positive for anxiety disorders, TTS was mainly triggered by a emotional stress; in patients without pre-existing anxiety disorders, undetermined event are more likely to trigger TTS; and physical triggers are more frequently associated with the second group but without statistically significant difference (Table 4) [29].

\begin{tabular}{|l|l|l|l|}
\hline TRIGGER & $\begin{array}{l}\text { PRE-ADMISSION ANXIETY DISORDER } \\
\text { PATIENTS }\end{array}$ & $\begin{array}{l}\text { PRE-ADMISSION WITHOUT ANXIETY DISORDERS } \\
\text { PATIENTS }\end{array}$ & $\begin{array}{l}\text { P- } \\
\text { VALUE }\end{array}$ \\
\hline $\begin{array}{l}\text { Emotional } \\
\text { stress }\end{array}$ & $74 \%$ & $30 \%$ & 0,001 \\
\hline $\begin{array}{l}\text { Physical stress } \\
\text { Undetermined }\end{array}$ & $16 \%$ & $37 \%$ & 0,070 \\
\hline
\end{tabular}

TABLE 4: Triggers in Patients With and Without Pre-Admission Anxiety Disorders

Table 5 mentions case-reports of patients with a diagnosis of Takotsubo syndrome and coexisting anxiety disorders, most of which were triggered by emotional, stressful events. 


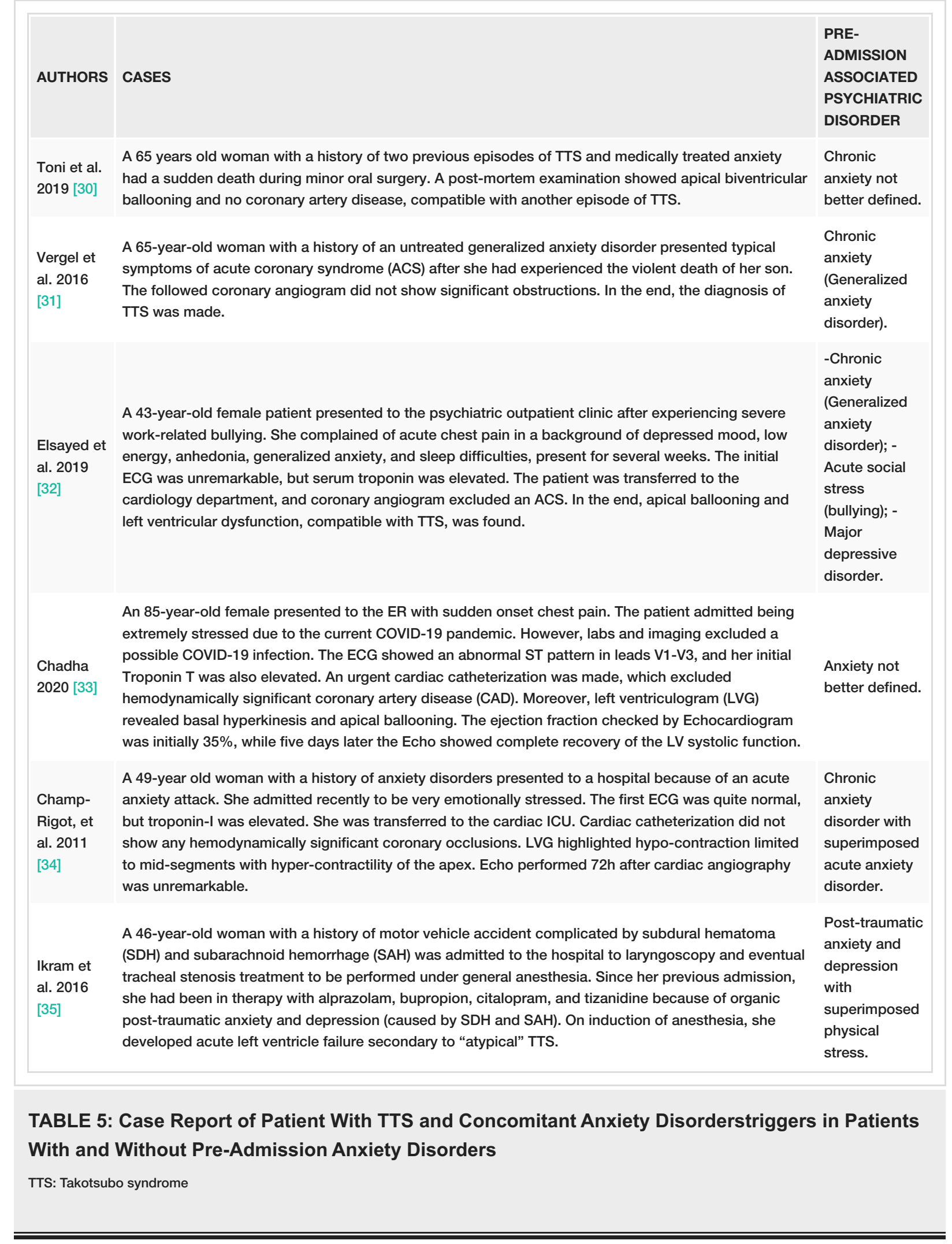

Takotsubo and Anxiety Disorders: Pathophysiologic Connections

Although the pathophysiology of TTS is not fully understood, much evidence has been documented. At the very beginning, spasms of coronary arteries were identified as TTS's underlying mechanism, although this hypothesis was later refuted [28]. However, it is well known that brain neurons that mediate vasoconstriction innervate coronary microcirculation, supports the concept that regional myocardial stunning among patients with TTS may be of neurogenic origin [22]. By the time, numerous studies demonstrated increased sympathetic activity, and consequent catecholamine excess, in TTS patients $[27,36]$. 


\section{Cureus}

The higher epinephrine levels stimulate the $\beta 2$-adrenoceptor coupling switch from membranous Gs to Gi proteins, with a consequent negative inotropic effect. It leads to cardiac protection because the "Gs->Gi switch" limits the degree of acute myocardial injury in response to the catecholamine excess [36,37]. Moreover, it was proven that the highest density of $\beta$-adrenergic receptors in the human heart is the apex [36]. Thus, this different anatomical distribution, coupled with the catecholamine over-release, could explain the characteristic "apical ballooning" of the majority cases of TTS. Patients affected by depression or anxiety disorders also show sympathetic overactivity in response to physical and emotional stress. Psychoneuro-endocrinological activation of the autonomic nervous system is expressed in two-stage responses [38]: I. acute response mediated by the activation of the central nervous systemadrenal-medulla axis, leading to catecholamine release in the adrenal medulla; II. chronic response caused by activation of hypothalamuspituitary-adrenal axis secondary to stressors, leading to cortisol release from the adrenal cortex. Indeed, the sympathetic system hyperactivity occurring in both TTS and anxiety disorders may in part explain the link between these conditions.

Furthermore, some authors have described a connection between TTS and inflammatory responses of the myocardium. In murine-based experiments, a predominant myocardial M1 macrophage infiltration (proinflammatory tissue destructive) was found in TTS, and no significant switch to M2 macrophages appeared (anti-inflammatory and profibrotic function) [39]. It has also been demonstrated that cytokines, which increase during inflammation state, indirectly increase the sympathetic output from central and peripheral autonomic nervous system nerve fibers and directly affect ion channels of cardiomyocytes. Specifically, interleukin (IL)- $1 \beta$ and IL- 6 enhance cardiac calcium channels, while tumor necrosis factor- $\alpha$ (TNF- $\alpha$ ) reduced the expression of potassium channels on myocardial cells [40]. The final effect of these ion current changes leads to electrocardiographic QTc prolongation, frequently found in TTS [4]. On the other hand, it has been verified that serum cytokine concentration is higher in many patients suffering from anxiety disorders [41].

We propose an integrated model with the aim of integrating the different studies concerning the pathophysiology of TTS together (Figure 1):

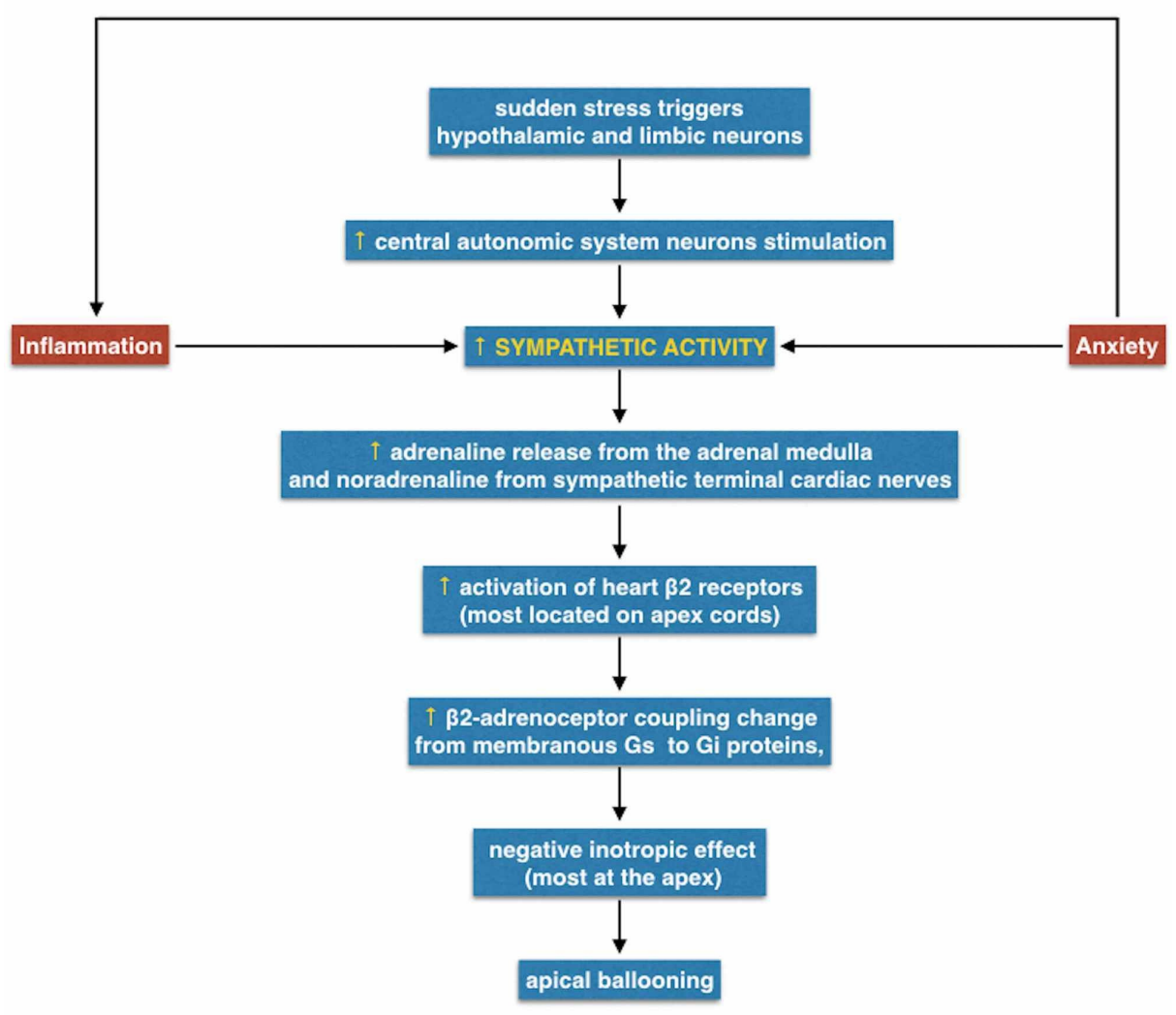

FIGURE 1: Takotsubo and Anxiety Disorders: Pathophysiologic

\section{Connections}

Psychoactive Drugs

Since a connection between anxiety disorders and TTS occurrence has been reported [22-25], it would seem 
conceivable that, by adequately treating anxiety disorders, we could reduce the incidence of TTS. Nevertheless, data available in the literature do not show univocal results.

For example, in a case report of a 67-year-old woman with a past medical history of anxiety and major depression disorders in treatment with clonazepam and fluoxetine (a selective serotonin reuptake inhibitor or SSRI), the tapering of her medication may have triggered an episode of TTS. Since she felt good and her mood had been stable at the time, her doctor decided to lower the dosage of anxiolytics. After three days, she developed chest pain, so she decided to go to the ER. After hospitalization, a diagnosis of TTS was made. Thus, the authors of this study hypothesized a protective role of anxiolytics in the development of TTS [42].

Another case report partially supports the above result. In this study, a postmenopausal woman with a history of TTS in preventive treatment with beta-blockers and ACE-inhibitors had a recurrence of Takotsubo syndrome six months from the previous diagnosis. She was subsequently discharged with sertraline (an SSRI) plus cognitive-behavioral therapy (CBT) without recurrent episodes during the following months. Indeed, the authors sustained that the combination of SSRI-CBT therapy should be a possible therapeutic aid in preventing TTS recurrence [43]. However, it should also be considered that in a significant retrospective multi-centered study, it has been evaluated that antidepressants, specifically SSRIs, were taken more frequently at admission in TTS patients than in ACS controls (respectively $17.1 \%$ vs $6.9 \%$ and $10.3 \%$ vs $3.4 \%$; p-value <0.001) [22]. Nevertheless, this result can be explained by looking at the greater prevalence of psychiatric diseases, primarily anxiety, in patients with TTS in this study. Moreover, to date, there have been no clear cases of SSRIs as triggers of TTS [44].

On the other hand, some authors consider the use of serotonin-norepinephrine reuptake inhibitors (SNRIs) could trigger TTS $[45,46]$. In a case report, a 52-year-old female, after being put on venlafaxine because of depression, developed TTS a few weeks later. She was discharged without venlafaxine, and, at the six-week follow-up visit, left ventricle kinesis and motion were unremarkable [45]. Again, in another case report, the use of duloxetine (another SNRI) seems to have been the trigger for a TTS episode [46]. The patient of this study, a 59-year-old woman with a history of depression, developed TTS after one week from the uppertitration of duloxetine. During the hospitalization, duloxetine therapy was discontinued. After one month from the acute event, the patient's left ventricular function recovered entirely without reoccurrence [46]. It may be plausible that the increased postsynaptic concentration of serotonin and norepinephrine caused by SNRIs could trigger TTS episodes. However, given the limited extension of these studies and the limited follow-up of the patients enrolled, it could be premature to suggest discontinuation of venlafaxine, duloxetine, or other SNRIs until new evidence confirms the results of the above studies.

Recurrence and Complications

Five-year recurrence rates occur in about 5-22\%, with the second episode occurring three months to 10 years after the first $[4,16]$. However, few studies have investigated the role of pre-existing psychiatric disorders as possible promoters of TTS recurrence. In a study conducted on 306 patients with TTS, one-third of whom had pre-existing psychiatric disorders, the TTS recurrence rate at follow-up was approximately $7 \%$. Notably, patients with pre-existing psychiatric illness were at higher risk of recurrent TTS. Nevertheless, from the same study, pre-existing psychiatric disorders were not associated with a more prevalent 30-day or long-term mortality [47].

Acute in-hospital complications are more prevalent in TTS patients with pre-existing psychiatric disorders. To demonstrate this, a retrospective analysis conducted on 455 TTS highlighted that presence of pre-existing psychiatric disorders as well as physical triggers, acute neurologic disorders, an elevated first troponin (more than 10 times the upper limit), and a reduced left ventricular ejection fraction of less than $45 \%$ were independent predictors for acute in-hospital complications. Authors included death from any cause, need for catecholamine use, cardiogenic shock, use of invasive or noninvasive ventilation, or cardiopulmonary resuscitation as acute in-hospital complications [22]. In another retrospective study, the authors calculated the ejection fraction (EF) recovery time in 36 TTS patients. The median recovery of EF was 25 days. Then they divided "early recoverers" patients whose EF returned to normal in less than or equal to 25 days (group 1) and "late recoverers" whose recovery needed more than 25 days (group 2). Demographic and clinical factors were compared between the groups. Generalized anxiety disorder was seen more commonly in the group with early recovery [48]. Thus long-term complications seem no to be affected by pre-existing psychiatric disorders $[47,48]$.

\section{Limitations}

Our review does not take into account articles published before 2010. Furthermore, we selected publications whose abstract was written in English. Unfortunately, many of the papers analyzed are based on case reports or retrospective case-controls with small sample sizes. While searching the literature, there were no specific clinical trials that deal with psychiatric disorders/anxiety disorders and Takotsubo syndrome. Moreover, by thoroughly reading each article involved, the diagnosis of anxiety disorders or depression was not established by using the same diagnostic criteria.

\section{Conclusions}


As many cases of TTS are triggered by emotional stress, we wondered if there was a higher prevalence of psychiatric disorders in these patients to exploit, in the absence of specific therapy, new therapeutic targets usable to prevent TTS onset and recurrence. The pre-admission prevalence of anxiety disorders is more prevalent in patients who developed TTS than in the healthy population and STEMI/ACS controls. On the other hand, we are confident to affirm that depression is not associated with a higher occurrence of TTS.

There is a robust pathophysiological interconnection between anxiety, inflammation, and TTS, leading to an increase in sympathetic activity. Patients with pre-admission psychiatric disorders have a higher risk of recurrent TTS and are more predisposed to suffer from acute in-hospital complications. The use of SSRIs, possibly in combination with CBT therapy, could be a potential therapeutic aid in preventing TTS's recurrence in selected patients. Since our review showed that anxiety disorders promote the onset and recurrence of TTS, a multidisciplinary approach, including psychiatrists or psychologists, may be highly desirable to provide the best care while reducing costs due to intra-hospital complications. Moreover, our review lays the foundations for possible new studies. For example, having documented a potential "protective role" of SSRIs, It would be useful to conduct a clinical trial where a group of patients with established anxiety disorders and TTS would receive beta-blockers (controls). In contrast, another group would be treated with beta-blocker plus SSRI.

\section{Additional Information \\ Disclosures}

Conflicts of interest: In compliance with the ICMJE uniform disclosure form, all authors declare the following: Payment/services info: All authors have declared that no financial support was received from any organization for the submitted work. Financial relationships: All authors have declared that they have no financial relationships at present or within the previous three years with any organizations that might have an interest in the submitted work. Other relationships: All authors have declared that there are no other relationships or activities that could appear to have influenced the submitted work.

\section{References}

1. Thygesen K, Alpert JS, Jaffe AS, et al.: Fourth universal definition of myocardial infarction. J Am Coll Cardiol. 2018, 72:2231-2264. 10.1016/j.jacc.2018.08.1038

2. Sharkey SW, Lesser JR, Maron MS, et al.: Why not just call it tako-tsubo cardiomyopathy: a discussion of nomenclature. J Am Coll Cardiol. 2011, 57:1496-1497. 10.1016/j.jacc.2010.11.029

3. Eitel I, von Knobelsdorff-Brenkenhoff F, Bernhardt P, et al.: Clinical characteristics and cardiovascular magnetic resonance findings in stress (Takotsubo) cardiomyopathy. JAMA. 2011, 306:277-286. 10.1001/jama.2011.992

4. Lyon AR, Bossone E, Schneider B, et al.: Current state of knowledge on takotsubo syndrome: a position statement from the taskforce on takotsubo syndrome of the heart failure association of the european society of cardiology. Eur J Heart Fail. 2016, 18:8-27. 10.1002/ejhf.424

5. Deshmukh A, Kumar G, Pant S, et al.: Prevalence of takotsubo cardiomyopathy in the United States . Am Heart J. 2012, 164:66-71. 10.1016/j.ahj.2012.03.020

6. Brinjikji W, El-Sayed AM, Salka S: In-hospital mortality among patients with takotsubo cardiomyopathy: a study of the national inpatient sample 2008 to 2009. Am Heart J. 2012, 164:215-221. 10.1016/j.ahj.2012.04.010

7. Summers MR, Prasad A: Takotsubo cardiomyopathy: definition and clinical profile. Heart Fail Clin. 2013, 9:111. 10.1016/j.hfc.2012.12.007

8. Scantlebury DC, Prasad A: Diagnosis of Takotsubo cardiomyopathy. Circ J. 2014, 78:2129-2139. 10.1253/circj.cj-14-0859

9. Citro R, Rigo F, D'Andrea A, et al.: Echocardiographic correlates of acute heart failure, cardiogenic shock, and in-hospital mortality in tako-tsubo cardiomyopathy. JACC Cardiovasc Imaging. 2014, 7:119-129. 10.1016/j.jcmg.2013.09.020

10. Redfors B, Vedad R, Angerås $\mathrm{O}$, et al.: Mortality in takotsubo syndrome is similar to mortality in myocardial infarction - a report from the swedeheart registry. Int J Cardiol. 2015, 185:282-289. 10.1016/j.ijcard.2015.03.162

11. Schneider B, Athanasiadis A, Schwab J, et al.: Complications in the clinical course of tako-tsubo cardiomyopathy. Int J Cardiol. 2014, 176:199-205. 10.1016/j.ijcard.2014.07.002

12. Schneider B, Athanasiadis A, Stöllberger C, et al.: Gender differences in the manifestation of tako-tsubo cardiomyopathy. Int J Cardiol. 2013, 166:584-588. 10.1016/j.ijcard.2011.11.027

13. Syed FF, Asirvatham SJ, Francis J: Arrhythmia occurrence with takotsubo cardiomyopathy: a literature review. Europace. 2011, 13:780-788. 10.1093/europace/euq435

14. Murakami T, Yoshikawa T, Maekawa Y, et al.: Characterization of predictors of in-hospital cardiac complications of Takotsubo cardiomyopathy: multi-center registry from Tokyo CCU network. J Cardiol. 2014, 63:269-273. 10.1016/j.jjcc.2013.09.003

15. Pant S, Deshmukh A, Mehta K, et al.: Burden of arrhythmias in patients with takotsubo cardiomyopathy (apical ballooning syndrome). Int J Cardiol. 2013, 170:64-68. 10.1016/j.ijcard.2013.10.041

16. Schultz T, Shao Y, Redfors B, et al.: Stress-induced cardiomyopathy in Sweden: evidence for different ethnic predisposition and altered cardio-circulatory status. Cardiology. 2012, 122:180-186. 10.1159/000338814

17. Santoro F, Ieva R, Musaico F, et al.: Lack of efficacy of drug therapy in preventing takotsubo cardiomyopathy recurrence: a meta-analysis. Clin Cardiol. 2014, 37:434-439. 10.1002/clc.22280

18. Goh AC, Wong S, Zaroff JG, et al.: Comparing anxiety and depression in patients with takotsubo stress 
cardiomyopathy to those with acute coronary syndrome. J Cardiopulm Rehabil Prev. 2016, 36:106-111. 10.1097/HCR.0000000000000152

19. Emdin CA, Odutayo A, Wong CX, et al.: Meta-analysis of anxiety as a risk factor for cardiovascular disease . Am J Cardiol. 2016, 118:511-519. 10.1016/j.amjcard.2016.05.041

20. Hare DL, Toukhsati SR, Johansson P, et al.: Depression and cardiovascular disease: a clinical review . Eur Heart J. 2014, 35:1365-1372. 10.1093/eurheartj/eht462

21. Allgulander C: Anxiety as a risk factor in cardiovascular disease . Curr Opin Psychiatry. 2016, 29:13-17. 10.1097/YCO.0000000000000217

22. Templin C, Ghadri JR, Diekmann J, et al.: Clinical features and outcomes of takotsubo (stress) cardiomyopathy. N Engl J Med. 2015, 373:929-938. 10.1056/NEJMoa1406761

23. Summers MR, Lennon RJ, Prasad A: Pre-morbid psychiatric and cardiovascular diseases in apical ballooning syndrome (tako-tsubo/stress-induced cardiomyopathy): potential pre-disposing factors?. J Am Coll Cardiol. 2010, 55:700-701. 10.1016/j.jacc.2009.10.031

24. Salmoirago-Blotcher E, Rosman L, Wittstein IS, et al.: Psychiatric history, post-discharge distress, and personality characteristics among incident female cases of takotsubo cardiomyopathy: a case-control study. Heart Lung. 2016, 45:503-509. 10.1016/j.hrtlng.2016.07.008

25. El-Sayed AM, Brinjikji W, Salka S: Demographic and co-morbid predictors of stress (takotsubo) cardiomyopathy. Am J Cardiol. 2012, 110:1368-1372. 10.1016/j.amjcard.2012.06.041

26. Del Pace S, Parodi G, Bellandi B, et al.: Anxiety trait in patients with stress-induced cardiomyopathy: a casecontrol study. Clin Res Cardiol. 2011, 100:523-529. 10.1007/s00392-010-0276-X

27. Zvonarev V: Takotsubo cardiomyopathy: medical and psychiatric aspects. role of psychotropic medications in the treatment of adults with "broken heart" syndrome. Cureus. 2019, 11:5177. 10.7759/cureus.5177

28. Buchmann SI, Lehmann D, Stevens CE: Takotsubo cardiomyopathy-acute cardiac dysfunction associated with neurological and psychiatric disorders. Front Neurol. 2019, 10:917. 10.3389/fneur.2019.00917

29. Lazzeroni D, Bini M, Castiglioni P, et al.: Anxiety disorders and stressful events in takotsubo syndrome . Cardiol J. 2018, 25:495-500. 10.5603/CJ.a2017.0136

30. Toni C, Iannaccone F, Chella P, et al.: Sudden death in a case of recurrent Takotsubo syndrome . Forensic Sci Med Pathol. 2019, 15:595-597. 10.1007/s12024-019-00163-w

31. Vergel J, Tamayo-Orozco S, Vallejo-Gómez AF, et al.: Síndrome del corazón roto y estrés agudo. a propósito de un caso acute stress and broken heart syndrome. a case report. Revista Colombiana de Psiquiatría. 2017, 46:257-262. 10.1016/j.rcp.2016.09.001

32. Elsayed, M., Connemann, et al.: Takotsubo cardiomyopathy with inconspicuous initial electrocardiogram: a potentially serious cardiac pathology related to emotional stress. Front Psychiatry. 2019, 10:308. 10.3389/fpsyt.2019.00308

33. Chadha S: 'COVID-19 pandemic' anxiety-induced takotsubo cardiomyopathy. QJM. 2020, 113:488-490. 10.1093/qjmed/hcaa135

34. Champ-Rigot L, Alexandre J, Grollier G, et al.: Atypical tako-tsubo syndrome: a morphologic variant or a step towards recovery?. Int J Cardiol. 2011, 146:256-258. 10.1016/j.ijcard.2010.10.068

35. Ikram S, Saleem N, Latif RK: Acute left ventricle failure on induction of anesthesia: a case report of reverse stress cardiomyopathy-presentation, diagnosis and treatment. J Anesth. 2016, 30:911-914. 10.1007/s00540016-2222-1

36. Ghadri JR, Wittstein IS, Prasad A, et al.: International expert consensus document on takotsubo syndrome (part I): clinical characteristics, diagnostic criteria, and pathophysiology. Eur Heart J. 2018, 39:2032-2046. 10.1093/eurheartj/ehy076

37. Paur H, Wright PT, Sikkel MB, et al.: High levels of circulating epinephrine trigger apical cardiodepression in a $\beta 2$-adrenergic receptor/Gi-dependent manner: a new model of takotsubo cardiomyopathy. Circulation. 2012, 126:697-706. 10.1161/CIRCULATIONAHA.112.111591

38. Kastaun S, Gerriets T, Tschernatsch M, et al.: Psychosocial and psychoneuroendocrinal aspects of takotsubo syndrome. Nat Rev Cardiol. 2016, 13:688-694. 10.1038/nrcardio.2016.108

39. Wilson HM, Cheyne et al.: Characterization of the myocardial inflammatory response in acute stressinduced (takotsubo) cardiomyopathy. JACC Basic Transl Sci. 2018, 3:766-778. 10.1016/j.jacbts.2018.08.006

40. Lazzerini PE, Capecchi PL, Laghi-Pasini F: Long QT syndrome: an emerging role for Inflammation and Immunity. Front Cardiovasc Med. 2015, 2:26. 10.3389/fcvm.2015.00026

41. Felger JC: Imaging the role of inflammation in mood and anxiety-related disorders . Curr Neuropharmacol. 2018, 16:533-558. 10.2174\%2F1570159X15666171123201142

42. Lafferty CJ, Lafferty KM, Bhat T, et al.: Takotsubo cardiomyopathy precipitated by tapering of anxiolytic medication: a case report. Clin Med Insights Cardiol. 2017, 11:1179546817746643. 10.1177/1179546817746643

43. Tunzi MA, Dinkha L, Sharp AJ, et al.: Recurrent takotsubo cardiomyopathy: getting to the root of the problem. Am J Case Rep. 2020, 21:923067. 10.12659/AJCR.923067

44. Nayeri A, Rafla-Yuan E, Krishnan S, et al.: Psychiatric illness in takotsubo (stress) cardiomyopathy: a review . Psychosomatics. 2018, 59:220-226. 10.1016/j.psym.2018.01.011

45. Vasudev R, Rampal U, Patel H, et al.: Selective serotonin-norepinephrine reuptake inhibitors-induced takotsubo cardiomyopathy. N Am J Med Sci. 2016, 8:312-315. 10.4103/1947-2714.187153

46. Selke KJ, Dhar G, Cohn JM: Takotsubo cardiomyopathy associated with titration of duloxetine. Tex Heart Inst J. 2011, 38:573.

47. Nayeri A, Rafla-Yuan E, Farber-Eger E, et al.: Pre-existing psychiatric illness is associated with increased risk of recurrent takotsubo cardiomyopathy. Psychosomatics. 2017, 58:527-532. 10.1016/j.psym.2017.04.008

48. Shaikh N, Sardar M, Jacob A, et al.: Possible predictive factors for recovery of left ventricular systolic function in Takotsubo cardiomyopathy. Intractable Rare Dis Res. 2018, 7:100-105. 10.5582/irdr.2018.01042 